\title{
Independent Faults in the Cloud
}

\author{
Rachid Guerraoui and Maysam Yabandeh \\ School of Computer and Communication Sciences, EPFL, Switzerland \\ firstname.lastname@epfl.ch
}

\begin{abstract}
Byzantine fault tolerant (BFT) protocols are replicationbased solutions to the problem of tolerating the arbitrary failures of software and hardware components. The essential assumption for replication is independence of failures. In this paper, we categorize four different failure independence levels that could be obtained from the cloud. Not surprisingly, providing more level of independence comes with the cost of more delays and less bandwidth. We report on our experiments to identifying the most appropriate BFT protocol for each level.
\end{abstract}

\section{Categories and Subject Descriptors}

C.2.4 [Computer-Communication Networks]: Distributed Systems-cloud computing; C.4 [Performance of Systems]: Fault tolerance

\section{General Terms}

Performance, Reliability, Security, Experimentation

\section{Keywords}

Byzantine fault tolerance, BFT, cloud computing, fault independence, consensus

\section{INTRODUCTION}

A software system is logically created of multiple layers, each layer implementing a particular functionality. Choosing the right layer for implementing a feature is a design decision, which could affect both the correctness and the performance of the software. End-to-end argument [10] highlights the drawbacks of an unnecessary implementation of a feature at lower layers: (i) not all the upper-layer modules might need the feature and yet have to pay for it; and (ii) the general implementation of the feature might not correctly satisfy the specific requirements of the upper-layer modules that are actually using it.

Permission to make digital or hard copies of all or part of this work for personal or classroom use is granted without fee provided that copies are not made or distributed for profit or commercial advantage and that copies bear this notice and the full citation on the first page. To copy otherwise, to republish, to post on servers or to redistribute to lists, requires prior specific permission and/or a fee.

LADIS'10 Zürich, Switzerland

Copyright 2010 ACM 978-1-4503-0406-1 ...\$10.00.

\section{Core Server Switch Data center OS Compiler Bugs}

Figure 1: The spectrum of failures.

Having end-to-end argument in mind, we can rethink the tolerance of Byzantine faults by BFT protocols [9, 3]: when should we use a separate BFT layer to tolerate faults? A recent study [12] lists some practical faults that could not be avoided by a naive BFT layer. Alternatively, the safety can be directly addressed by the application, e.g., Google file system [5] leaves the unexpected duplicate records in the files to be dealt by upper-layer applications separately. BFT protocols target tolerating arbitrary, independent faults. In other words, as long as occurrences of a given fault are independent, a BFT protocol can tolerate them. Otherwise multiple manifestations of the fault at the same time could violate the service correctness. Plainly, a BFT protocol cannot tolerate unknown, arbitrary faults since it is impossible to ensure independence of a fault that it is still unknown. Therefore in practice, to correctly tolerate faults with BFT (i) the set of faults must be specified, and (ii) the deployment setup must be engineered to provide independence for those faults.

The spectrum of faults, as depicted in Figure 1, can range from the failure of a core inside the processing unit, to programming errors. Although the genuine independence is not achievable in reality, depending on the kind of fault, the level of independence can be improved by using some specific replication settings. The more we move towards the programming errors direction, the harder it is to provide failure independence. To have real failure independence for programming errors we need multiple independent developing teams, which is very expensive, whereas a simple replication over some servers inside a local area network (LAN) is enough to provide core failure independence. In other words, the independence of manifestations of a fault comes with a price, and not all the application, hence, might be willing to pay for that cost. Following end-to-end argument, we should verify whether (i) the deployment setup can correctly deliver independence of the faults, and (ii) the particular applications of interest can afford the expenses of independence.

As a first step of the pragmatic approach in using BFT, this paper categorizes the range of faults, for which the independence can be cheaply delivered by the current state of technology. For each category, which provides independence for only a particular set of faults, we then reevaluate the state-of-the-art BFT protocols. For cheap fault indepen- 
dence we, in particular, consider the cloud, as a promising, growing business model that can help reduce the IT costs. ${ }^{1}$

Two general groups of failures are considered: (i) hardware failures, which include faulty core, power failure, storage failure, and failure of intermediate switches and routers; and ii) malicious attacks, which include security attacks that compromise the virtual machine (VM) or the entire server, ${ }^{2}$ as well as Denial of Service (DoS) attacks that can make a server, switch, or router to saturate and become unavailable. To tolerate hardware failures, the service should be replicated on independent hardware components. The cloud provider can increase the independence of the involved hardware components by assigning customer nodes to different (i) machines, (ii) LANs, and (iii) geographically distributed data centers.

The attacker that compromises some replicas inside a cloud could use them as agents of a distributed DoS attack. Therefore, replicas located on the same cloud have a similar risk of failure by a DoS attack. Besides, using multiple clouds could increase the independence of software vulnerabilities if the cloud vendors use separate software components, e.g., the host operating system and the virtualization technology. Nevertheless, after a replica is compromised, the replication factor as well as the address of the other replicas can be obtained by the attacker who then can focus its attacks on them, either by trying to compromise them or by directing DoS attacks towards them. To achieve more independence of replica failures, the address of the other replicas must be unknown to a (potentially compromised) replica. We refer to this technique as oblivious clouds.

After a brief overview of BFT protocols in Section 2, we categorize the replication setups into four groups: (i) multiple machines inside a LAN, (ii) separate LANs (multiple availability zones), (iii) separate cloud providers, and (iv) oblivious clouds; the Byzantine faults in each category will be correctly tolerated, assuming existence of only a subset of faults. Different system characteristics of the replication setups, such as latency and bandwidth, demand different BFT protocols. Using experimental results from bftsim [11], we highlight the appropriate BFT protocol for each category.

\section{BACKGROUND}

BFT protocols are replication-based solutions to the problem of tolerating arbitrary failures of software and hardware components. A BFT protocol can ensure safety and progress of up to $f$ of a particular faulty components, if more than $3 f$ replicas of that component are used in replication. For example, if the application is replicated on four separate machines, then the BFT protocol can tolerate at most one faulty hard disk [2]. In this section, we give a brief overview of BFT protocols as well as different BFT setups. The BFT protocols differ in the number of required phases to commit (communication rounds), response latency, and throughput. In general, there is a trade-off between latency and throughput; to have high throughput, the contention between two competing client requests must be avoided by using a primary. The primary orders the client requests and then forwards the ordered requests to the other replicas. Although

\footnotetext{
${ }^{1}$ Note that launching BFT protocols is a client-side decision and the cloud vendor does not have to be involved in it.

${ }^{2} \mathrm{~A}$ physical machine in the cloud is shared between multiple clients via virtualization.
}

this offers high throughput, the commit latency increases because of the extra phase of communicating through the primary.

To tolerate malicious attacks, the messages must be authenticated via some cryptographic techniques, such as Public Key Cryptography (PKC), which authenticates a single message, and Message Authentication Code (MAC), which authenticates a single channel (and its messages). PKC could make the BFT protocols much simpler since it is verifiable even after the message is forwarded multiple times, but it is around 100 times slower than MAC. The throughput could be bounded by the number of MAC operations per request performed by the bottleneck replica. This is mostly the case in $0 / 0$ setup, where $q / p$ stands for requests and replies with payload size of $q$ and $p \mathrm{~KB}$, respectively.

For large message sizes ( $4 / 4$ benchmark), the throughput is bounded by the input/output bandwidth of the bottleneck replica, i.e., the replica that sends/receives more messages per request. An example of such a bottleneck is multicasting the request by the primary. The multicast cost can be remedied in setups such as LAN that support hardware multicast. Nevertheless the cloud vendors might be reluctant in offering the hardware multicast support due to its scalability issues [13].

In PBFT [3], the client sends the request to all the replicas including the primary. The primary determines a sequence number and forwards the order to other replicas. To detect the faulty primary, all the replicas broadcast the received order to ensure that other replicas have received the same order for the request. Then all the replicas broadcast the ordered request and execute it. This phase is necessary to detect the interference of two primaries, which might happen during view change. The client accepts the replies if they match.

In Zyzzyva [8], the client sends the request only to the primary. However, after other replicas receive the request as well as its sequence number from the primary, they immediately execute it and send the reply to the client. The client accepts the replies if they all match. Otherwise, either the primary or some of the replicas are faulty. In this case, the first correct client can detect that and demand changing the primary.

Chain [6] also uses a primary to avoid contention. All other replicas are ordered as a chain and each one forwards the request to the next. The last replica sends the reply to the client. This technique increases the end-to-end delay, but the throughput improves as the number of MAC operations by each replica is close to 1, i.e. the theoretical lower bound. The key idea is to partition the replicas into two groups, where one group only verifies the client requests and the other only authenticates the reply. After detecting the failure, the whole protocol aborts and the abort history is used to initialize another instance of BFT protocol. This technique is called Abortable BFT [6].

Protocols that use a primary can take advantage of a technique named batching; the primary batches $N$ requests (mostly $N=10[3,8]$ ) and performs the MAC operation only once on all of them. The increased latency is negligible in high-throughput systems. Q/U [1] does not use a primary and the clients directly communicate with the replicas. If two clients are accessing the replicas at the same time, the protocol requires more number of phases to resolve the contention. Although it has the advantage of optimal communi- 


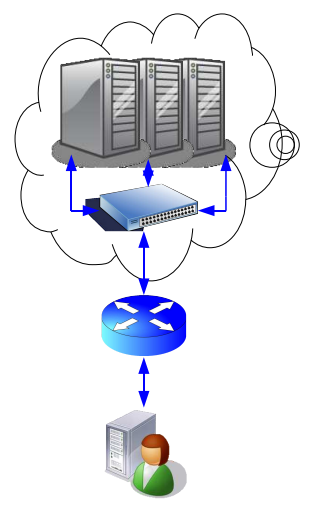

Figure 2: The LAN setup.

cation rounds in non-contention cases, it requires more number of replicas to operate, i.e., $5 f+1$. Moreover, increasing the number of clients could increase the contention. Quorum [6], an abortable version of $\mathrm{Q} / \mathrm{U}$ that uses less number of replicas $(3 f+1<5 f+1)$, also suffers from the contention problem.

\section{MULTIPLE MACHINES}

A LAN setup, in which the replicas are connected by a switch, offers the best performance for replication because of the low latency (1 ms) and high bandwidth (1 Gbps) between the replicas. The clients of the cloud are always connected through a wide area network (WAN), which implies 10-30 ms latency and higher packet loss rate. The setup is depicted in Figure 2.

On the other hand, the LAN setup offers the lowest failure independence among the possible setups we consider. Upon failure of the switch connecting the replicas, the protocol cannot progress any more. Furthermore, a LAN can spread only over a limited local area. It is likely, therefore, that some failures, such as the main power failure, a fire, and an earthquake, affect all the replicas. In the particular case that the VMs are placed inside the same physical machine or rack, the failures are even more dependent since the failure of the physical machine or the rack power source, respectively, will affect all the replicas.

In theory, the BFT protocols are usually compared based on the required number of phases to commit a value. In practice, however, the low latency of the LAN makes the difference between the overall latency of the BFT protocols negligible. Instead, leveraging the high available bandwidth of LAN becomes more appealing. For small payloads $(0 / 0$ benchmark) where the bottleneck is the CPU, Chain [6] is the best candidate since it performs only $1+\epsilon$ MAC operations; the $\epsilon$ parameters gets closer to zero, by using higher degree of batching.

Figure 3 compares the throughput of the state-of-the-art BFT protocols (excluding chain), using bftsim. The batching factor is always 10 and is specified by a "b10" suffix. The timeout values in bftsim are set to $0.204 \mathrm{~s}, 0.210 \mathrm{~s}$, and $0.206 \mathrm{~s}$ for Q/U, PBFT, and Zyzzyva, respectively. The client-replica and replica-replica latency is set to $60 \mathrm{~ms}$ and $0.08 \mathrm{~ms}$, respectively. In all the experiments, the loss rate of client-replica communications is set to $0.05 \%$, client-replica bandwidth is $1 \mathrm{Mbps}$, and the number of clients is chosen

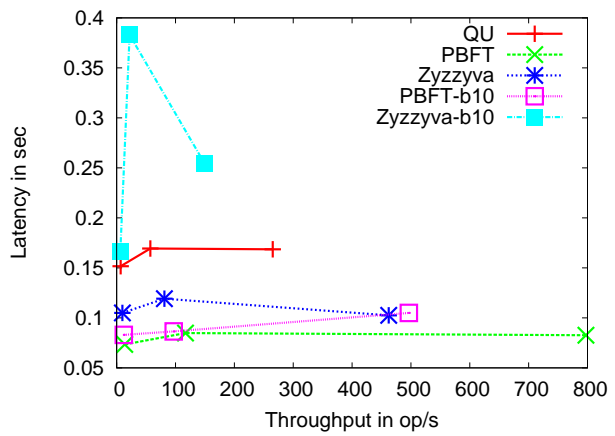

Figure 3: 0/0 benchmark, same LAN.

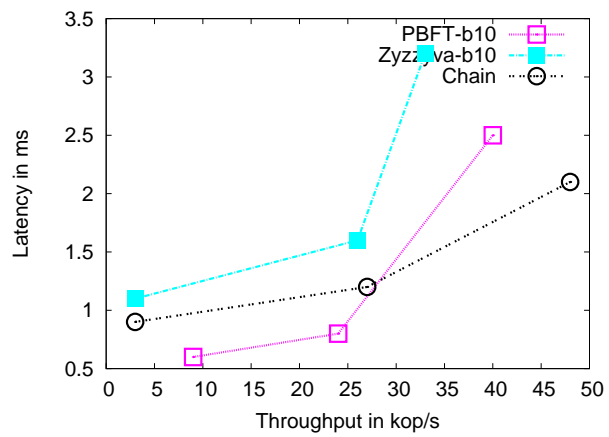

Figure 4: 0/0 benchmark [6].

among 1, 10, and 50. As we expected, the low latency of LAN, makes PBFT have the best performance. Even for a few clients that probability of contention is low, $Q / U$ has higher commit latency than PBFT. This is counter-intuitive since PBFT requires some more replica-replica transmissions to commit a value. This can be explained by the fact that $\mathrm{Q} / \mathrm{U}$ requires receiving multiple client messages by separate replicas, whereas PBFT requires delivering only one client message to the primary for order assignment. It is shown that in high variance of WAN, waiting for more messages increases the overall latency [7].

Since we did not have any implementation of Chain in bftsim, we use the reported numbers at the related work [6], to compare Chain with the other protocols. Figure 4 shows that Chain outperforms all the other protocols in high throughputs. Because the Chain uses fewer client-replica messages compared to PBFT and Zyzzyva, we expect that the same conclusion applies to the setup where the clients are behind a WAN.

Figure 5 presents the experimental results of $4 / 4$ benchmark. ${ }^{3}$ The change in the message size does change the performance, but PBFT is still leading. Zyzzyva performs worse than PBFT, since it is more sensitive to the replicaclient message delay and loss; not receiving all the replies before timeout, makes Zyzzyva to fall into a two phase recovery operation. We could not justify the appeared knee in

\footnotetext{
${ }^{3}$ The missing numbers in the figures imply that the corresponding experiments never finished. This is either a liveness bug in the protocol or a problem in the bftsim implementation. In the case of Zyzzyva, we observed a similar behavior in the experiments with the $\mathrm{C}++$ implementation released by its authors.
} 


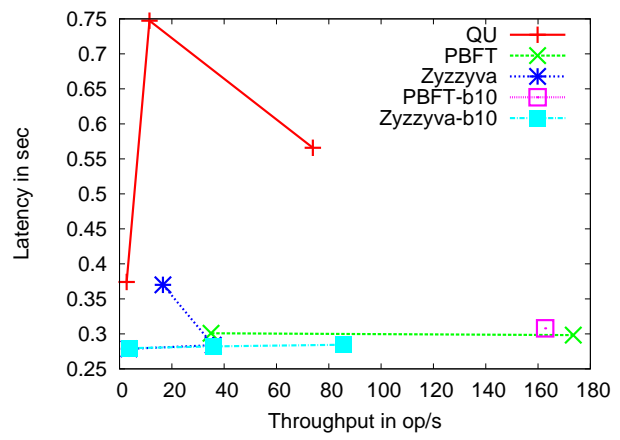

Figure 5: 4/4 benchmark, same LAN.

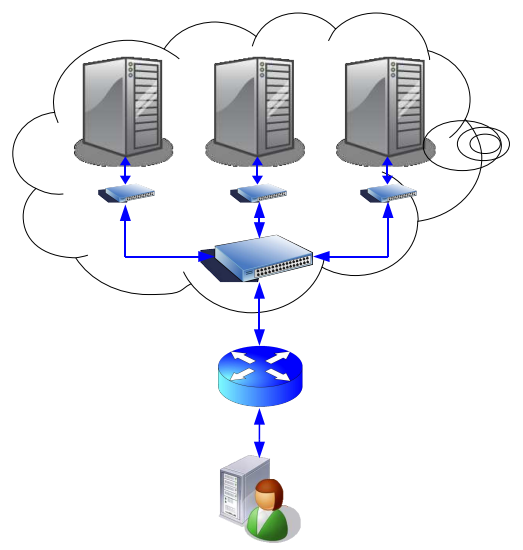

Figure 6: The setup for multiple availability zones.

the performance of $\mathrm{Q} / \mathrm{U}$ by increasing the number of clients. The same pattern was observed in Figure 8 of bftsim simulation results [11], but that also was left unexplained.

We also ran experiments having multicast enabled for both $0 / 0$ and $4 / 4$ benchmarks. Surprisingly, the throughput of the protocols dropped after enabling multicast. Since the multicast feature in bftsim is simulated by computing less transferring delay, we could not interpret the drop in the throughput.

\section{MULTIPLE AVAILABILITY ZONES}

Availability zones are distinct locations inside the same geographical region that are engineered to have independent failures via separate power-lines, being isolated in separate rooms not to allow a fire to propagate to them, and probably using separate gateway routers. ${ }^{4}$ The setup is depicted in Figure 6. Because they are located in the same region, the latency between them is still low $(1-5 \mathrm{~ms})$ and the available bandwidth is still high (100-1000 Mbps). The higher latency makes the BFT protocols with less number of required phases more appealing. Multicast feature might be available (although less likely) depending on the engineering of the zones.

Figures 7 and 8 compare the performance of the protocols in $0 / 0$ and $4 / 4$ benchmarks, respectively. The timeout values in bftsim are set to $0.212 \mathrm{~s}, 0.242 \mathrm{~s}$, and $0.222 \mathrm{~s}$ for

\footnotetext{
${ }^{4}$ Since Amazon does not offer this information, we do not know how isolated the availability zones in EC2 [4] really are.
}

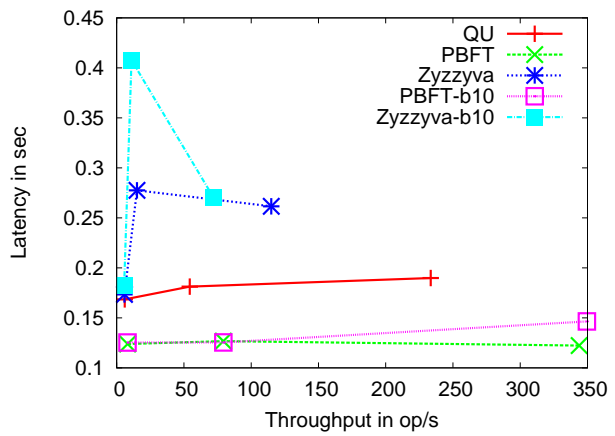

Figure 7: 0/0 benchmark, multiple zones.

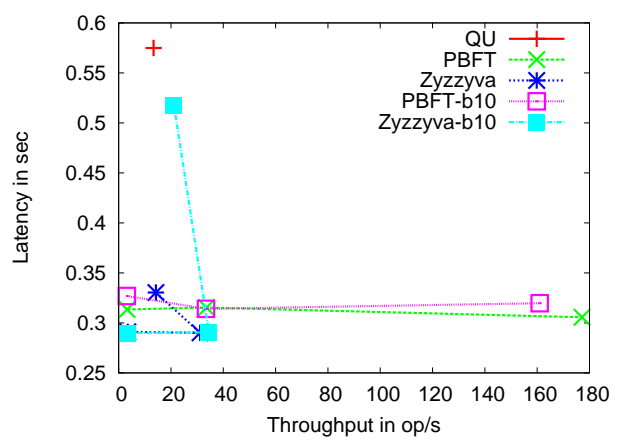

Figure 8: 4/4 benchmark, multiple zones.

Q/U, PBFT, and Zyzzyva, respectively. The client-replica and replica-replica latency is set to $60 \mathrm{~ms}$ and $10 \mathrm{~ms}$, respectively. The replica-replica bandwidth is set to 1 Gbps. Both the throughput and the latency of Zyzzyva-b10 are improved by increasing the number of clients from 10 to 50, perhaps because the increase in number of requests makes the batching technique more effective. Again, the sensitivity of Zyzzyva to the WAN delays makes the PBFT to be still the best option. However, for low number of clients in 4/4 benchmark, Zyzzyva outperforms PBFT. It can be explained by the fact that in PBFT the clients have to send the requests to all the replicas, while in Zyzzyva it is sent to only the primary. For large messages, this overhead can have a negative impact on PBFT's performance. For larger number of clients, Zyzzyva becomes over-saturated and we observe a decrease in throughput as well as an increase in latency. The over-saturation had also been observed in other experiments with bftsim [11].

\section{MULTIPLE CLOUD PROVIDERS}

To provide failure independence against regional power failures and disasters, such as an earthquake or a tsunami, the replicas must be placed over separate geographical regions. Geographical distribution of replicas also provides availability against tier-1 router failures. Amazon already offers computing units distributed over three separate regions. However, separating cloud providers also offers failure independence against vulnerabilities of a particular cloud provider. For example, if the replica maintained by a cloud provider is compromised because of vulnerability in the used VM technology, the other replicas can still progress since the 


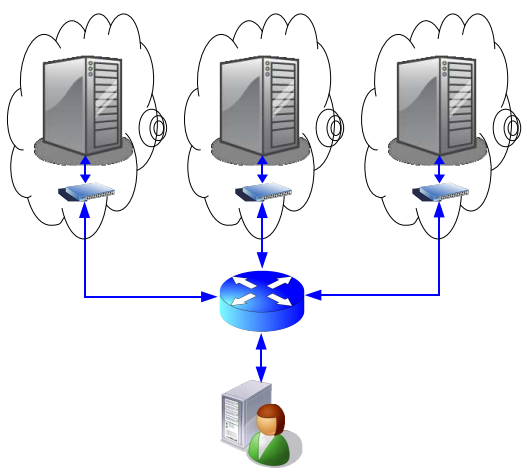

Figure 9: The setup for multiple clouds.

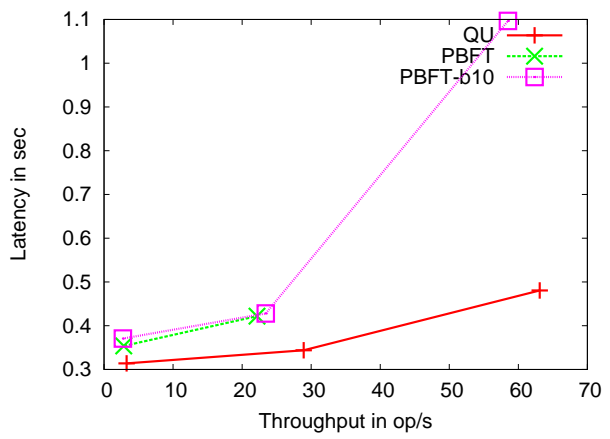

Figure 10: 0/0 benchmark, multiple clouds.

corresponding cloud providers probably use a different technology for vitalization.

The setup is depicted in Figure 9. The WAN that connects the clouds has high latency (10-30 ms) and low bandwidth (1-10 Mbps). Moreover, the multicast feature is not available, which has a negative impact on the performance of protocols such as PBFT and Zyzzyva. Not existence of multicast increases the overhead on the primary, which is in charge of sending the proposals to all replicas.

Figures 10 and 11 compare the performance of the protocols in $0 / 0$ and $4 / 4$ benchmarks, respectively. The timeout values in bftsim are set to $0.262 \mathrm{~s}, 0.442 \mathrm{~s}$, and 0.322 s for Q/U, PBFT, and Zyzzyva, respectively. The clientreplica and replica-replica latencies are set to $60 \mathrm{~ms}$. The replica-replica bandwidth is set to $1 \mathrm{Mbps}$. The loss rate of replica-replica communications is set to $0.05 \%$. The high latency between the replicas, make the performance of PBFT and Zyzzyva to drop very quickly. In contrary, the $\mathrm{Q} / \mathrm{U}$ 's performance surprisingly scales very well with the number of clients. This is in contradiction with the intuition that more clients increases the contention in the $\mathrm{Q} / \mathrm{U}$ and hence lowers the throughput. We can explain this as following: because of the high WAN delays, the throughput is in general low anyway, and hence the interference between the client's requests occurs rarely in $\mathrm{Q} / \mathrm{U}$.

\section{OBLIVIOUS CLOUDS}

Although the distribution of the service over multiple could providers offers a high availability against non-malicious attacks, the failure of a compromised replica will still increase the odds of failure of other replicas. In other words, the

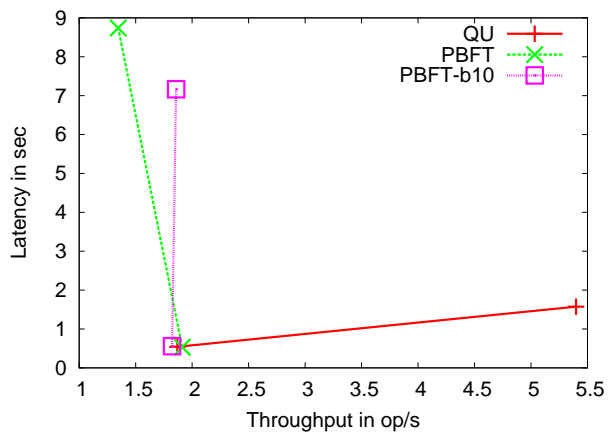

Figure 11: 4/4 benchmark, multiple clouds.

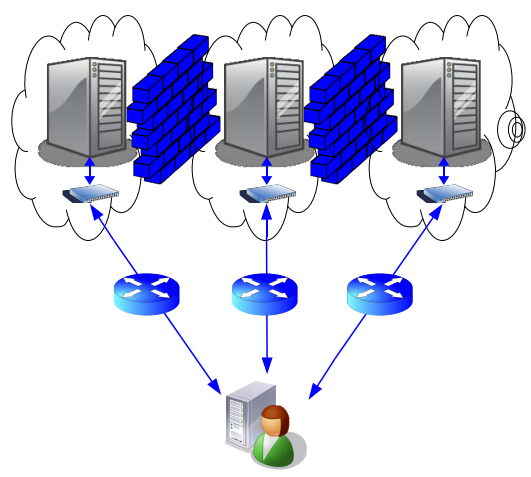

Figure 12: Oblivious clouds setup.

failures of replicas are not completely independent. The reason is that after a replica is compromised, the location of the other replicas can be obtained from the compromised replica. ${ }^{5}$ The attacker can then focus its attacks on other replicas. For example, it is very difficult to avoid a distributed DoS attack when the attacker has enough motivation to do so and also knows the location of the target victim.

To keep the address of the replicas anonymous, there should not exist any replica-replica communication. Therefore, all communications must be performed through the clients. The setup is depicted in Figure 12. In this scheme, the cloud providers (and consequently the replicas) do not even know that they are running a replication protocol. The BFT protocols that fall into this category are Q/U [1] and Quorum [6]. It is worth noting that $Q / U$ specification allows replica-replica communication for object synchronization. Hence, to be able to use $\mathrm{Q} / \mathrm{U}$ in the oblivious clouds setup, the corresponding optimization in $\mathrm{Q} / \mathrm{U}$ must be disabled.

\section{CONCLUSION}

This paper, categorizes the different levels of failure independence that can be offered to BFT protocols by different setups in the cloud. Providing higher failure independence comes with the performance penalty due to the increased latency and the decreased bandwidth. Because the clients are typically connected via a WAN, the protocols that are sensitive to the replica-client delay and loss do not perform

\footnotetext{
${ }^{5} \mathrm{An}$ attacker that is based inside the cloud can also obtain this information by monitoring the network traffic of a replica.
} 
well. Chain achieves the best throughput when the replicas are connected via a LAN. For availability zones, where the latency between the replicas is higher, PBFT offers the best performance. Q/U performed the best for the setup that the replicas are geographically distributed, because of using no communication between the replicas. To achieve the highest

failure independence in the oblivious clouds setting, the only available options are Q/U and Quorum.

\section{ACKNOWLEDGMENTS}

We thank Nikola Knežević for useful discussions.

\section{REFERENCES}

[1] M. Abd-El-Malek, G. Ganger, G. Goodson, M. Reiter, and J. Wylie. Fault-scalable Byzantine fault-tolerant services. ACM SIGOPS Operating Systems Review, 39(5):59-74, 2005.

[2] G. Bracha and S. Toueg. Asynchronous consensus and broadcast protocols. Journal of the ACM (JACM), 32(4):824-840, 1985.

[3] M. Castro and B. Liskov. Practical Byzantine fault tolerance and proactive recovery. ACM Transactions on Computer Systems (TOCS), 20(4):398-461, 2002.

[4] Amazon ec2. http://aws.amazon.com/ec2/.

[5] S. Ghemawat, H. Gobioff, and S. Leung. The Google file system. ACM SIGOPS Operating Systems Review, 37(5):43, 2003.

[6] R. Guerraoui, N. Knežević, V. Quéma, and M. Vukolić. The next 700 BFT protocols. In Proceedings of the 5th European conference on Computer systems, pages 363-376. ACM, 2010.

[7] F. Junqueira, Y. Mao, and K. Marzullo. Classic paxos vs. fast paxos: caveat emptor. In Proceedings of the 3rd USENIX/IEEE/IFIP Workshop on Hot Topics in System Dependability (HotDep.07). Citeseer, 2007.

[8] R. Kotla, L. Alvisi, M. Dahlin, A. Clement, and E. Wong. Zyzzyva: speculative byzantine fault tolerance. ACM SIGOPS Operating Systems Review, 41(6):45-58, 2007.

[9] L. Lamport. Time, Clocks, and the Ordering of Events in a Distributed System. Com. of the $A C M$, 21(7):558-565, 1978.

[10] J. Saltzer, D. Reed, and D. Clark. End-to-end arguments in system design. ACM Transactions on Computer Systems (TOCS), 2(4):288, 1984.

[11] A. Singh, T. Das, P. Maniatis, P. Druschel, and T. Roscoe. BFT protocols under fire. In Proceedings of the 5th USENIX Symposium on Networked Systems Design and Implementation, pages 189-204. USENIX Association, 2008.

[12] Y. Song, F. Junqueira, and B. Reed. BFT for the skeptics (WIP). In SOSP, 2009.

[13] Y. Vigfusson, H. Abu-Libdeh, M. Balakrishnan, K. Birman, R. Burgess, G. Chockler, H. Li, and Y. Tock. Dr. multicast: Rx for data center communication scalability. In Proceedings of the 5th European conference on Computer systems, pages 349-362. ACM, 2010. 